\title{
Ejercicio de la ciudadanía global femenina ante los tribunales internacionales: el impacto del activismo ciudadano en la corte interamericana de derechos humanos
}

\author{
Nina Ferrer Araújo" \\ Recibido: abril de 2015 \\ Evaluado: junio de 2015 \\ Aprobado: noviembre de 2015
}

\section{RESUMEN}

El documento asume que el ejercicio de la ciudadanía plena, en un contexto globalizado, debe partir de consideraciones políticas, culturales y económicas antes que de un reconocimiento legal. Se entiende que la ciudadanía global, como un vehículo planetario, puede ser ostentada por personas individuales y por grupos identitarios, como las mujeres, de manera que se llega a concebir el movimiento social de mujeres como el Estado en Red de Castells. Sin embargo, se aprecia que este ejercicio ciudadano no puede ser pleno, sin la existencia de unos tribunales internacionales de justicia que garanticen los derechos implícitos en el concepto de ciudadanía, que en el caso concreto de la Corte Interamericana ha venido moldeando el ejercicio de la ciudadanía global a partir del establecimiento de unos estándares jurisprudenciales en materia de derechos humanos de las mujeres, aplicables en cada uno de los Estados que han reconocido la competencia de la Corte Regional.

Palabras clave: ciudadanía femenina, ciudadanía global, derechos humanos de las mujeres y derechos de las mujeres en el Sistema Interamericano.

\footnotetext{
El presente texto constituye parte de los resultados del Proyecto de investigación en curso "Ejercicio de la Ciudadanía Global femenina ante los Tribunales Internacionales" a cargo de la autora, que inició el día 21 de julio de 2014; adscrito a la línea de investigación Sistema Jurídico y Sociedad del grupo de investigación DEMOSOPHIA de la Facultad de Derecho y Ciencias Políticas de la Universidad San Buenaventura Cartagena.

** Abogada, especialista en Derecho Comercial de la Universidad Libre de Cartagena, candidata a magíster de la Facultad Latinoamericana de Ciencias Sociales (FLACSO), en espera de titulación. Investigadora del Grupo de Investigaciones DEMOSOPHIA de la Facultad de Derecho y Ciencias Políticas de la Universidad San Buenaventura Cartagena, en la línea de investigación Sistemas Jurídicos y Sociedad. Docente de dedicación de la misma Facultad. Asociada también al Grupo de investigaciones Sociales y Jurídicas del Programa de Derecho de la Fundación Universitaria Tecnológico Comfenalco en Convenio con la Universidad de Medellín, docente catedrática de tal Institución. Temática de investigación: "El acceso a la justicia como condición para el ejercicio de la ciudadanía femenina". Correo electrónico: nina.ferrer@usbctg.edu.co
} 


\title{
Exercise of Women's Global Citizenship before International Courts: Impact of Citizen Activism on the Inter-American Court of Human Rights
}

\begin{abstract}
This document assumes that the exercise of full citizenship, within a global context, should be based on political, cultural, and economic considerations rather than on a legal recognition. It is understood that global citizenship, as a world vehicle, can be exercised by individuals and identity groups such as women, in such a way that women's social movement can be seen as Castells' network society. It can be seen, however, that this exercise cannot be exercised in full without the existence of international justice courts that can assure rights implied in the concept of citizenship that, in the case of the Inter-American Court, has been setting the exercise of global citizenship from some jurisprudential standards on women's human rights, applicable to each State that has accepted the competence of the Regional Court.
\end{abstract}

Key words: Women's citizenship; global citizenship; women's human rights; women's rights in the Inter-American System. 
La ciudadanía es, sin lugar a dudas, uno de los pilares de la democracia; el grado de ejercicio de la ciudadanía es un indicador de la profundidad de esta en los Estados contemporáneos. Como concepto jurídico político ha sufrido grandes transformaciones de la mano del proceso histórico del sujeto activo que la posee; no obstante, siempre se ha concebido como un estatus de derechos que implica la inclusión de "unos", pero al mismo tiempo la exclusión de "otros", partiendo de la definición de quienes integran el "todos" y de la lucha de los "otros" por ostentar el estatus. Por lo anterior, se puede afirmar que han surgido ciudadanías emergentes, producto de tensiones sociales, políticas y jurídicas con el propósito de la inclusión.

Bajo el concepto de ciudadanías emergentes encaja el concepto de la femenina, de reconocimiento tardío e incompleto, que primero se manifestó desde lo cultural para más tarde lograr reconocimiento político y consecuentemente protección jurídica en el interior de los Estados, pero también ante la comunidad internacional, razón por la cual existe un ejercicio de ciudadanía global femenina. Es precisamente este aspecto el que ha dado origen al Proyecto de investigación en curso, denominado: "Ejercicio de la ciudadanía global femenina ante los tribunales internacionales de derechos humanos", del que surge el presente texto, cuyo propósito general es describir la manera como la Corte Interamericana de Derechos Humanos se ha convertido en espacio de ejercicio de ciudadanía global femenina, coadyuvando al posicionamiento de una agenda internacional de reivindicaciones de derechos humanos de las mujeres, como grupo identitario, pero también como individuos en contextos y circunstancias diferentes. Para tales fines se pretende dar respuesta a los siguientes interrogantes que guían el discurso: 1. ¿Cuáles son las diferentes dimensiones del proceso globalizador? 2. ¿Cómo se ha formado el concepto de ciudadanía femenina en lo local y nacional? Y 3. ¿De qué manera la Corte Interamericana de Derechos Humanos ha permeado el activismo social en pro del reconocimiento internacional de los derechos de las mujeres como ciudadanas plenas?

El Proyecto en mención se desarrolla desde la perspectiva holística de investigación fundamentada por la Fundación Sypal'; en tal sentido no presenta la

Según Jaqueline Hurtado (2000) en su libro Metodología de investigación holística, el holotipo de investigación analítico documental corresponde al criterio de fuente, caracterizado por la recuperación de la información documental y el análisis de contenido en función del evento criterio de análisis. Incluye el análisis de libros, escritos, videos, grabaciones etc. El círculo holístico que se diseñó y ejecutó en el Proyecto fue: 1. Fase Previa de Investigación que culmina con la determinación del enunciado holopráxico que condujo a la pregunta de investigación y al desarrollo del sintagma gnoselogógico que aterriza en la elaboración del Marco Teórico y finalmente el diseño metodológico inicial. 2. Fase de elaboración de la matriz de análisis, a partir del enunciado holopráxico, se construyó la matriz de análisis de las categorías previamente identificadas: ciudadanía femenina, acceso a la justicia de las mujeres, ciudadanía global y acceso a la justicia internacional en Derechos Humanos. 3. Fase de Recolección de Información: Esta fase está constituida por dos momentos, el inicial de revisión bibliográfica y el segundo de aplicación de la matriz de análisis. 4. Fase de Análisis, integración y presentación de resultados a partir de la cuál nace el artículo que se presenta en esta ocasión. 
visión tradicional de enfoques, sino que se sitúa en un holotipo de investigación denominado analítico-documental, que como su nombre lo indica se caracteriza por la recuperación de la información documental y el análisis de contenido en función del criterio de análisis que, en este caso, es el ejercicio de la ciudadanía femenina ante los tribunales internacionales de derechos humanos.

Finalmente, resta aclarar que el documento continúa la idea de investigación recurrente de la autora, que establece la conexidad entre el acceso a la justicia y el ejercicio de la ciudadanía, pero esta vez desde la ciudadanía global. Inicia, por lo tanto, con un acercamiento a la noción de globalización para abordar las distintas dimensiones que esta puede manifestar y específicamente su relación con la idea de una democracia y de ciudadanía. Las generalizaciones aterrizan desde una perspectiva de género en el análisis de la construcción de la ciudadanía global de las mujeres y la importancia que los tribunales de justicia tienen para el ejercicio de la misma.

\section{MOVIMIENTOS, FLUJO E INTERCAMBIOS DE LA GLOBALIZACIÓN}

Hablar de globalización es referirse a un proceso complejo, pluricausal en el que concurrieron una serie de fenómenos y circunstancias históricas, que se desdobla en el ámbito económico, cultural, político, jurídico científico-tecnológico y social. Dicho fenómeno tiene la particularidad de generarse a escala mundial, mientras que se mimetiza en lo local; quiere decir esto que, si bien parece un fenómeno de una dimensión extraña a la organización de los Estados nacionales, en realidad los transforma: el concepto de la soberanía nacional ha mutado sin dejar de existir, es decir, que tiene distintos modos de expresión según los contextos en los que se materialice y los actores que participen (Bonder, 2010).

Para intentar una definición, en principio es necesario decir que la globalización es en sí misma movimiento (Bauman, 1999), flujos e intercambios de fronteras, espacios, territorios, culturas, tradiciones, personas y capitales. Estos intercambios se dan a través de relaciones de poder (Bonder, 2010), pero entendiendo el poder desde una perspectiva distinta, ya no en cabeza de los entes estatales sino de los sujetos internacionales de poder, sobre los que volveremos más adelante.

Este constante movimiento y flujo explica una de las grandes características de la globalización: la creciente comunicación e interdependencia de los distintos países del mundo que concurren en un mercado global para generar intercambios. Así las cosas, en palabras de Manuel Castells (Castells, s. f.), es una transformación multidimensional definida por la alteración del sistema productivo, organizativo, cultural e institucional.

\subsection{Las "globalizaciones" o dimensiones de la globalización}

Como se mencionó, la globalización es multidimensional, un proceso de flujos e intercambios variados, en los ámbitos 
económicos, culturales, sociales y políticos. Hablamos de una globalización económica, en la que se ha creado un mercado financiero global, caracterizado por el desarrollo de unos productos muy sofisticados; la interdependencia de los mercados internos de cada país; la dependencia de todas las economías a sus empresas; el desarrollo intensivo del comercio internacional; la fortaleza de organismos financieros y comerciales internacionales (FMI, BIC y OMC); el impulso de la inversión extranjera, y el triunfo de las multinacionales.

Si bien es un fenómeno multilateral, cada una de las dimensiones de la globalización afecta tanto a los Estados como a cada uno de los individuos que hacen parte de ellos; el ser humano de hoy está situado al mismo tiempo en un ámbito local y en uno global. Las condiciones locales se traspasan a las globales, motivo por el cual los grupos segregados del Estado nación se constituyen también en grupos segregados en cada una de las dimensiones de la globalización, razón por la cual es importante la conformación de verdaderos movimiento sociales que interactúen a fin no solo de disminuir los efectos negativos de la globalización, sino de constituir los espacios globales, como espacios de profundización de las democracias locales.

La primera dimensión y la más evidente es la globalización económica que se caracteriza más por el consumo masivo de bienes y servicios que por la producción, determinando la existencia de un mapa social, en el que claramente se vislumbran dos clases sociales: los grandes consumidores y los que no pueden consumir, los de arriba y los de abajo (Bauman, 1999), los pobres y los ricos. Los de arriba son quienes se benefician de la ruptura espacio-tiempo producto de la globalización, mientras que los de abajo (generalmente grupos tradicionalmente excluidos: pobres históricos, mujeres, indígenas, afrodescendientes) padecen sus más crudos efectos.

Es de agregar que la transformación económica ha generado un mapa laboral nuevo, en el que, sin lugar a dudas, podemos hablar de un mercado laboral globalizado, donde fenómenos como el dumping social y la flexibilidad se han convertido en la cotidianidad, así como la internacionalización de los sindicatos. Lo dicho claramente altera y se proyecta en lo social, y repercute de manera especial en la vida de las mujeres que, en razón al reconocimiento incompleto de su ciudadanía nacional, son más vulnerables a padecer los efectos negativos de la globalización; por ejemplo, en aras de la producción vertiginosa, la maternidad y el cuidado de los menores no compartido en el interior de las familias se vislumbran como pérdida de competitividad. De igual forma, los trabajos flexibles son también herramientas para aumentar labor por menos costos, al mismo tiempo que mantiene a las mujeres cerca del hogar y de todas las tareas domésticas.

En cuanto a la dimensión cultural de la globalización, debe reconocerse previamente que se trata de un proceso 
generado por el intercambio y flujo físico de personas a través de migraciones, impulso de turismo a gran escala y comercio internacional entre otras situaciones, pero también por las comunicaciones. Quizá de este origen procede lo paradójico de esta dimensión que, por un lado, pone a conversar diversas identidades (hibridación), pero, por el otro, tiende a la homogeneización de las tradiciones.

Hablar de globalización cultural significa localizar lo global o glocalizar (Robertson, citado por Bonder, 2010), esto es, transformar las dinámicas locales en la cultura global. En este sentido, refleja, por una cara, la hibridación cultural en la que no es posible hablar de una identidad pura, ya que se presenta el diálogo intercultural, y por la otra, la pretensión de construcción de una cultura global homogénea que imponga estándares muchas veces ajenos a la tradición local, pero considerados como necesarios por la comunidad global que se corresponden en mayor medida con la cultura de los países de arriba.

Si reconocemos que existe un juego global de poder, aceptamos que existen instituciones, jerarquías y sujetos de poder, dentro de los que encontramos la ciudadanía, los grupos y movimientos sociales, las multinacionales o corporaciones, las instituciones y organizaciones mundiales, los tribunales internacionales y, lógicamente, el Estado-nación.

\subsection{De la globalización política en particular: La profunda levedad de una democracia global}

La globalización es, sin duda, un proceso político, dentro de la esfera de decisión de los Estados nacionales más poderosos y de las organizaciones financieras multilaterales (Ferrer, 1998). Vislumbra un cambio en el concepto de poder político, desde el tradicional poder monocéntrico, que reside en los Estados nación hacia uno policéntrico que reside en la comunidad política internacional (Rosenus, citado por Bonder, 2010). Lo anterior no quiere decir que los Estados nacionales hayan muerto; afirmar tal cosa sería desconocer que la globalización también tiene una faceta interna, en la medida que la mayoría de los procesos globales se materializan en espacios locales, luego de debates y consensos (Sassen, s. f.). De hecho, la mayor descentralización de los Estados nacionales, la privatización y la participación ciudadana en la formulación de políticas públicas son muestras de esta nueva conceptualización y de la adaptación del Estado-nación a este sistema. Se trata de la coexistencia de dos ámbitos de poder extremadamente interrelacionados: uno interno o local y otro externo, supranacional o global.

La transformación del concepto de poder político conduce a la incapacidad de la democracia actual para responder efectivamente a las demandas de justicia social en un mundo completamente polarizado (Bonder, 2010). En este orden de ideas, debe intentarse un modelo de democracia más flexible que implica un 
replanteamiento de la soberanía nacional, un reconocimiento de intereses y agendas comunes y globales - derechos humanos e internacional humanitario, problemas ambientales, fundamentalismo, discriminación de género etc.-, sin sacrificar los intereses específicos de los Estados y de ciertos grupos y movimientos plurilaterales, los mínimos de justicia global y la participación de los diversos actores en el reparto igualitario de las oportunidades, a fin de garantizar la gobernanza global legítima (Bonder, 2010).

La democracia global exige actores e instituciones globales, esto es, por un lado, partidos políticos globales (Beck, 2001), ciudadanos globales y por otro instituciones democráticas globales y Estados nacionales transformados en unos Estados en red, que funcionan mediante la interacción de sus distintos componentes, una malla de colaboración y de aplicación conjunta de los recursos que, como se mencionó anteriormente, se descentraliza, reconoce mayor autonomía regional y más participación formal de la ciudadanía en la toma de decisiones, a la postre que se abre a diversas formas de cooperación internacional, articulando los fines sociales del Estado con la actuación de las organizaciones no gubernamentales (Castells, s. f.).

\section{ACERCAMIENTO A LA NOCIÓN DE CIUDADANÍA EN UNA DEMOCRACIA GLOBAL}

El concepto teórico tradicionalmente desarrollado de ciudadanía se presenta siempre vinculado con la existencia de un Estado-nación. Recurrentemente se acude a T. H. Marshall a fin de delimitar el significado actual del vocablo ciudadanía, autor que desde 1949 intentó construir una noción más comprensiva de las diferentes relaciones que puede desarrollar el ciudadano con el Estado, en la que asoció la ciudadanía a una plenitud de derechos y a unos organismos propios vinculados al ejercicio concreto de la misma. Definió la ciudadanía como el máximo estatus reconocido por el Estado a sus asociados y la dividió en tres: política, económica y civil, e identificó para cada ámbito unos derechos e instituciones (Marshall, 1998).

El fenómeno de la globalización pone a tambalear la noción estricta propuesta por Marshall, debido a ella parte de la existencia de un Estado nación tradicional y del reconocimiento de derechos que este Estado les hace a los ciudadanos, así como de las diversas relaciones que surgen entre este y aquellos. La noción de ciudadanía global desafía la elaboración conceptual tradicional, y definiciones como la de "derecho a tener derechos", de Ana Arendt, alcanzan mayor nivel de significación (citado por Bareiro, 2009).

Del concepto de ciudadanía se pueden identificar dos elementos: por una parte, el ejercicio material de la misma, como componente dinámico, y, por la otra, el reconocimiento como componente institucional del Estado que goza de protección por parte del sistema jurídico. A partir de esta dicotomía entre la institucionalización y el ejercicio material de la ciudadanía, es más fácil iniciar la construcción del concepto de ciudadanía glo- 
bal, porque la ciudadanía global empieza a construirse desde lo político y cultural antes que desde lo jurídico (Vargas, 2001).

Según Virginia Vargas (2001) teniendo como base las variadas definiciones de ciudadanía, es posible establecer unos rasgos comunes, que vendrían a constituir la esencia del concepto: reconocimiento de la historicidad en la construcción de la noción; la aparente igualdad de derechos; la pertenencia del ciudadano a una comunidad política; una garantía jurídica del ejercicio de derechos; la existencia de un espacio público para su ejercicio y que genera siempre la dinámica de inclusión/ exclusión.

Los mencionados rasgos comunes pueden abordarse desde el ámbito global que se pretende: la ciudadanía global es una categoría histórica sin definición final o acabada, relativa a diversas relaciones existentes entre los sujetos de poder y la comunidad política global a la que pertenecen y en la que actúan, que se manifiesta en el reconocimiento de una gama de derechos denominados humanos, que cuentan con diversas formas de protección que incluyen la existencia de tribunales, así como la posibilidad de iniciar acciones jurisdiccionales.

De lo dicho podemos inferir que, así como iniciamos hablando de una dualidad de poder, o de la existencia de un fuero interno y otro externo del poder político, existen paralelamente dos ciudadanías, una política nacional y otra globalizada, ambas recaen sobre la persona individual (seres humanos), aunque la ciudadanía global parece extenderse a otros entes distintos, como se verá en adelante.

\subsection{Distintos marcos interpretativos en torno a la ciudadanía global}

Stromquist (2009), en su texto Teorizando la ciudadanía global: discursos, desafíos e implicaciones para la educación, ha agrupado los diferentes discursos teóricos que justifican la existencia de la ciudadanía global, en cuatro categorías, resumidas a continuación:

Teorías del realismo de la Nueva Era. Considera que apoyar la ciudadanía global es retornar a las misiones civilizadoras, se trata de la creación de una ciudadanía dentro del contexto del neoimperialismo. Esta corriente asume a Estados Unidos de América como un actor global clave en la construcción del orden global, categoría que alcanza su mayor influencia en la política exterior norteamericana y la política antiterrorista, que parte de la idea de la existencia de unos Estados delincuentes y de otros apaciguadores del orden mundial, apoyados en organismos internacionales como la ONU, la OMC, y el Banco Mundial.

Es obvio que a partir de esta noción claramente jerárquica y excluyente no puede construirse una definición de ciudadanía global acorde con el concepto de democracia global.

Teoría de la ciudadanía corporativa. Expresa la idea de asimilar las corporaciones a nuevos ciudadanos, reconocidos como centros de poder económico y político. 
Actualmente a las transnacionales, se les están concediendo una serie de prerrogativas legales a partir de las políticas desarrollistas de la OMC y el Banco Mundial, que simultáneamente conceden poder a los países desarrollados en detrimento de los tercermundistas. Las corporaciones son ciudadanos privilegiados, en la medida que poseen múltiples nacionalidades, lo que implica obviamente múltiples ciudadanías estatales.

Por lo anterior, esta corriente considera que las verdaderas ciudadanas globales son las corporaciones, constituyéndose esta afirmación en el principal peligro de esta teoría, en la medida que transforma un concepto jurídico y político en otro completamente económico y desnaturalizado.

Teorías de la cultura mundial. Trátese de una visión sociológica, basada en el reconocimiento de una cultura global diversa, que observa patrones educativos abiertos al aprendizaje multicultural; enfatiza los derechos humanos como valor supremo, y en este sentido reconoce la labor de las ONG como herramientas de difusión de estos valores cosmopolitas. Concibe entonces unos seres pertenecientes a una comunidad política global, en la cual están llamados a ejercer su ciudadanía.

Mantiene que en el desarrollo de la ciudadanía intervienen dos dinámicas complementarias: el aprendizaje de los derechos y las obligaciones y el desarrollo de marcos institucionales democráticos. Es en este aspecto donde esta teoría ha sido atacada, por la dificultad práctica de la constitución de un único gobierno global.

Teorías del vehículo planetario. Esta óptica de interpretación nace de los grupos comunitarios. Reconoce que el aumento de la movilidad de productos y personas implica que las políticas públicas deben enfocarse en los problemas globales, que afectan especialmente a grupos históricamente subordinados como las mujeres y los pueblos indígenas.

Desde esta visión, las organizaciones no gubernamentales (ONG) y los movimientos sociales se convierten en ciudadanos que ejercen presión sobre los gobiernos para lograr la solución a esos problemas globalmente definidos. Hacen parte de estos temas trasnacionales: el asunto ambiental, el fundamentalismo, el racismo, la interculturalidad, el terrorismo y, sobre todo, la reivindicación de los derechos humanos, específicamente de las mujeres y de la población LGBTI.

El activismo de estos grupos, se ha fortalecido exponencialmente, gracias a la facilidad de la transmisión de información y al desarrollo de redes que las tecnologías de información y comunicación permiten. No obstante, el activismo y las comunicaciones son solo un aspecto del ejercicio de la ciudadanía, y en ocasiones no son suficientes para alcanzar el poder de influenciar la toma de decisiones, y por ello se hace necesario el establecimiento de un sistema coercitivo trasnacional.

Es esta visión de ciudadanía global la que podría considerarse más acertada, 
puesto que parte no solo de la existencia de una cultura global, sino también de unos problemas globales, reconoce como sujetos de poder global tanto a las personas individualmente consideradas, a los Estados y a organismos internacionales, como a las ONG y los movimientos sociales trasnacionales; estos dos últimos, además, se comportan como verdaderos ciudadanos globales, imitando el rol de las personas individuales.

\subsection{La ciudadanía femenina de lo nacional a lo global}

La ciudadanía nacional, desde el concepto marshalliano tradicional, es una moneda de dos caras: una de ellas es la reconocida igualdad, pero la otra es una exclusión con pretensión de legitimarse; en efecto, si la ciudadanía es un estatus reconocido a algunos de los asociados de un Estado, iguala a quienes ostentan la categoría, pero excluye a quienes no la tienen. La femenina se caracteriza por una institucionalización tardía; desde sus orígenes en la polis fue un estatus que no se reconoció a la mujer, igualmente, en la Modernidad, quedaron fuera del contrato social, por existir un contrato sexual concomitante que subordinaba a las mujeres (Pateman, 2002).

En consecuencia, la ciudadanía de las mujeres es una emergente, cuyo reconocimiento va de la mano de los procesos de democratización de los derechos humanos, producto de las gestas de las mujeres organizadas como uno de los "Nuevos Movimientos Sociales", que según Delgado Salazar (2007) constituyen un conjunto de formas de acción colectiva, diferentes a aquellas basadas en las clases, cuyos propósitos marcados se encuentran en la reivindicación de los derechos de grupos poblacionales tradicionalmente marginalizados, tales como afrodescendientes, LGBTI, indígenas, personas con discapacidad, mujeres, entre otros.

Además de recibir una institucionalización tardía, la ciudadanía nacional femenina, una vez reconocida, es limitada, en la medida que las mujeres permanecen relegadas a las relaciones domésticas familiares; así lo explican Pateman (2002) y Judith Astelarra (2002), quienes manifiestan que la ciudadanía nacional plena podrá alcanzarse en la medida que se instituyan nuevos modelos de familia, no solo que modifiquen las relaciones entre hombres y mujeres, sino que incorporen en el ámbito de lo público los derechos reproductivos y los derechos a que la ciudadanía reciba los cuidados personales necesarios para su supervivencia.

Esta deficiencia en la institucionalización de la ciudadanía nacional femenina históricamente impulsó a que las mujeres, excluidas formalmente del Estado-nación como sujetas políticas, encontraran alternativas distintas a su ejercicio democrático, esto es, que la ciudadanía femenina en lo local primero se ejerció y luego se reconoció: nació de lo cultural, antes que de lo jurídico, tal como ocurre con la ciudadanía global, lo que explicaría la forma contundente en que ha llegado a ejercerse.

La organización del movimiento de mujeres en el interior de los Estados nacio- 
nales a fin de lograr mayor impacto se ha preocupado por la articulación en redes y grupos trasnacionales, la participación en conferencias y foros internacionales, la apertura a las nuevas tecnologías de información, la movilidad de saberes, el reconocimiento de una agenda trasnacional y de unos temas comunes como, por ejemplo, la violencia basada en género; los derechos sexuales y reproductivos, el reforzamiento de identidades globales -mujeres indígenas, afro, lesbianas entre otras-, de tal manera que reproduce el ciclo. La ciudadanía global femenina fue un proceso en principio cultural, que más tarde tuvo reconocimiento político y que hasta hace poco se ha ido acercando al goce jurídico.

\section{LOS TRIBUNALES INTERNACIONALES DE JUSTICIA Y LA CIUDADANÍA GLOBAL}

Desde diferentes perspectivas el concepto de ciudadanía siempre aparece ligado al otorgamiento de derechos subjetivos, como facultades reconocidas a determinadas personas. En algunas ocasiones, para ejercer sus derechos quienes son titulares deben acudir a los tribunales como espacio propio de los derechos en disputa, de manera que el acceso a la Administración de Justicia es un derecho, pero al mismo tiempo también puede ser visto como una herramienta de efectividad de todos los implícitos en el concepto de ciudadanía.

En otras palabras, de igual manera que el acceso a la justicia es un requisito indispensable para el ejercicio ciudadano en el espacio doméstico, que solo a través de la existencia de unos tribunales justos, imparciales, autónomos y eficientes, puede el Estado contemporáneo garantizar el ejercicio pleno de los derechos y por lo tanto el ejercicio pleno de la ciudadanía (Ferrer, 2010), los tribunales internacionales son elementos indispensable para la democracia global y por lo tanto para el ejercicio de la ciudadanía global.

Así las cosas, los derechos humanos se transforman en el núcleo esencial de la ciudadanía, en la medida que son un conjunto de facultades reconocidas a todos los seres humanos, pero, a su vez, el reconocimiento de los derechos humanos (DDHH) por parte de la comunidad internacional fuerza históricamente la creación de unas instituciones globales de protección y garantía de los mismo, que suplan aquello casos en los que los Estados de manera interna han permitido la violación de los DDHH, o que no contemplan formas jurídicas de protección de los mismos.

En relación con los derechos humanos, es preciso acotar que los movimientos de mujeres han influenciado dramáticamente en la evolución de los mismos desde dos aspectos: el primero consistente en el reconocimiento de las mujeres como sujetas de derechos humanos en condiciones de igualdad, pero con necesidades específicas de protección, tales como el derecho a vivir una vida libre de violencias, el derecho al cuerpo (sexuales), derechos reproductivos, estabilidad laboral de la mujer embarazada, entre otros; segundo, percibir los derechos humanos más allá 
del ámbito jurídico como una cultura de los derechos humanos, a partir de los avances recogidos en las convenciones, pactos y programas de acción de Naciones Unidas:

Se genera en las prácticas de los movimientos un proceso de reconceptuación de los derechos humanos que amplía su alcance desde el ámbito de los Estados, en su doble calidad de garantes y/o violadores de los derechos humanos, a todos los ámbitos de la vida cotidiana, tanto públicos como privados. Con ello se modifica la percepción del Estado como el único violador de los derechos humanos y compromete tanto a los Estados como a los individuos. Se requiere para su protección de un rol más activo en la difusión de los derechos entre todos los miembros de la sociedad en una cultura que desde la familia y la educación ponga el acento en el valor y el respeto integral del hombre y la mujer (Fries, 2000, p. 52).

\subsection{Ejercicio ciudadano de las mujeres ante los tribunales internacionales}

Debido a las tensiones que históricamente han marcado el camino de la construcción de la ciudadanía femenina, puede observarse que el movimiento social de mujeres es uno de los grupos identitarios con más trabajo y reconocimiento al nivel global, en la medida que ha logrado construir agendas comunes, de manera que hoy existen asuntos de reivindicación de derechos de las mujeres aplicables a todas y también un reconocimiento de la importancia de trabajar por las necesidades de las otras. Así, por ejemplo, si bien es imperativa la defensa del derecho de las mujeres a una vida libre de violencias, las organizaciones de nivel mundial, a partir de la denuncia de los hechos de violencia basada en género en Juárez, exigieron el respeto del derecho a la vida y a la integridad sexual de las mujeres en ese territorio mexicano.

No obstante, el ejercicio de la ciudadanía por parte de las mujeres no solo se ha limitado al activismo tradicional, sino que ha penetrado en los tribunales de justicia internacionales, quienes a partir de la década de los 90, de manera paulatina, han ido desarrollando una consciente perspectiva de género en sus decisiones.

En este recorrido se hará énfasis en el Sistema Interamericano por cuestiones de extensión; no obstante, es menester referirse a tres decisiones básicas de los tribunales penales de Ruanda y la antigua Yugoslavia, por el importante precedente que marcaron en relación con la libertad sexual y la violencia sexual ejercida contra las mujeres como un acto de tortura y un delito de lesa humanidad. Al respecto, se ha entendido que la violencia sexual contra las mujeres es un tema global porque es una de las armas de guerra más extendidas en los conflictos armados contemporáneos. Su eficacia como instrumento de terror tristemente ha justificado su presencia en contextos afectados por violencia armada y política de todo el planeta; no obstante, solo a partir de la década de los 90 ha sido puesto por 
primera vez como asunto público tras la investigación y esclarecimiento de los hechos ocurridos durante el genocidio de Ruanda y los conflictos étnicos en la zona balcánica de Europa, durante los procesos que se llevaron a cabo ante los tribunales creados para ello (Villelas 2010, citando a Skjelsbaek, 2001)

El primero de los tres casos representativos es el de Dusko Tadic, quien fue condenado, por el Tribunal Penal para la antigua Yugoslavia, a 20 años de prisión el 14 de julio de 1997, en razón a que, durante el genocidio de la zona balcánica europea, inició una campaña de terror sistemática y organizada a través de diversas acciones de violencia. Se comprobaron casos de acceso carnal violento y actos sexuales abusivos, cometido en las prisiones de Omarska y Trnojpolje, contra mujeres bosnio-croatas y bosniomusulmanas (Mantilla E Uprimny, 2009). La importancia de este caso radica en el reconocimiento que efectuó el Tribunal de la violencia a sexual, como una de las múltiples formas a través de las cuales se puede infundir terror en la sociedad civil durante el conflicto, por lo que se constituyeron en actos de lesa humanidad.

El mismo Tribunal, en el caso Foca, luego de escuchar el testimonio de 25 mujeres, el 22 de febrero de 2001, condenó a tres hombres bosnio-serbios. Zoran Vukovic, Radomir Kovac y Dragoljub Kunarac a 12, 20 y 28 años de prisión, respectivamente, por delitos sexuales cometidos contra estas mujeres, perpetrados con el objetivo de provocar confesiones. En esta oportunidad, el Tribunal fue enfático al considerar la violencia sexual como una forma específica de tortura, y como delito de lesa humanidad; de igual manera consideró el control sexual como una forma de esclavitud (Mantilla E Uprimny, 2009).

Finalmente, el tercer caso es el Caso Akayesu, en el que el Tribunal de Ruanda, por primera vez en el ámbito internacional, relacionó la violencia sexual con el genocidio, siempre que esta se cometiere con el propósito específico de destruir total o parcialmente un grupo determinado como ocurrió con las mujeres Tutsis durante el conflicto étnico de Ruanda. El día 2 de septiembre de 1998, el Tribunal condenó a Jean Paul Akayesu, antiguo alcalde de Taba, por las múltiples violaciones a mujeres tutsi, además del asesinato de más de 2000 personas de esta etnia. A pesar de que Akayesu no forzó a ninguna mujer, sí permitió que sucedieran todos estos actos, llegando incluso a impulsar a sus subordinados a cometerlos.

\subsubsection{Ejercicio de la ciudadanía global ante la Corte Interamericana}

El Sistema Interamericano, a partir de 1994 con la adopción de la Convención de Belem Do Pará y la creación de la Relatoría Especial de la Comisión sobre los Derechos de la Mujer, bajo el mandato de analizar la observancia de las obligaciones establecidas en la Convención Americana de Derechos Humanos, ha dado pasos cualitativamente importantes en materia de protección de los derechos de las mujeres de la región, estableciendo unos 
estándares de protección que fueron recogidos de manera muy integral en el informe de la Comisión Interamericana de Derechos Humanos denominado "Estándares jurídicos vinculados a la igualdad de género y a los Derechos de las Mujeres en el Sistema Interamericano de Derechos Humanos; Desarrollo y Aplicación".

En el mencionado documento, más que recopilar los estándares, se pretende valorar el avance e implementación de las recomendaciones y la jurisprudencia emanadas del Sistema por parte de los Estados que han suscrito la Convención Americana de Derechos Humanos, así como la Convención de Belém Do Para; no obstante, inicia por una recapitulación de los estándares a analizar en el informe, que podrían ser resumidos así: existe un vínculo estrecho entre la violencia basada en género y la discriminación, que es un asunto sistemático y recurrente en la vida de las mujeres latinoamericanas, y no un caso aislado, que puede constituirse en actos de tortura; cuando se presenten casos de este tipo de violencia el Estado tiene la obligación de actuar con la debida diligencia requerida para prevenir, investigar, y sancionar con celeridad y sin dilación; deberá, además, disponer de mecanismos judiciales efectivos, adecuados, e imparciales para víctimas de violencia contra las mujeres; además, persiste la responsabilidad del Estado de implementar acciones para erradicar la discriminación contra la mujeres y los patrones estereotipados de comportamiento que promueven su tratamiento inferior en sus sociedades; estas acciones van desde el tema de adoptar políticas hasta el análisis y evaluación de las normas y prácticas judiciales (Comisión Interamericana de Derechos Humanos -OEA-, 2011).

No obstante, antes de pasar al análisis pormenorizado de los fallos de la Corte Interamericana en los que de manera emblemática se revelan estas pautas, es necesario acotar el principal límite de este tribunal internacional, que si bien parece fuerte por el reconocimiento que tiene su jurisprudencia en los sistemas jurídicos domésticos, encuentra debilidad en el hecho de que solo algunos instrumentos alcanzan el grado de protección regional; estos son: Convención Americana de Derechos Humanos (Pacto de San José de Costa Rica de 1969), la Convención Americana para Prevenir y Sancionar la Tortura de 1985 y la Convención Interamericana sobre Desaparición Forzada de Personas de 1994, El Protocolo Adicional a la Convención Interamericana en materia de Derechos Económicos, sociales y Culturales, pero sólo en los relacionado con los artículos 8 (derechos sindicales) y 13 (derecho a la educación) y la hoy todavía discutida competencia de la Corte en tratándose de la Convención de Belem Do Pará para prevenir, sancionar y erradicar la violencia contra la Mujer, en relación con el artículo 7 de la misma (Acosta, 2009).

En este último aspecto, relativo a la Competencia de la Corte para conocer de los casos de violación de los derechos contenidos en la Convención de Belém Do Pará, se ha entablado una discusión en relación con el alcance de los artículos 
11 y $12^{2}$ de la misma, en la medida que ninguno de ellos deja claro si puede o no la Corte Interamericana conocer de casos contenciosos por violación del Tratado. Precisamente esta fue la base de la excepción de falta de competencia ratione materiae del Estado mexicano en el caso "Campo Algodonero" en la que el mismo Tribunal se adjudicó competencia de manera contundente con las siguientes palabras: "En conclusión, una interpretación sistemática de las normas relevantes para resolver esta controversia permite respaldar aún más la competencia contenciosa de la Corte respecto al artículo 7 de la Convención Belém do Pará3".

2 Artículo 12 : Cualquier persona o grupo de personas, o entidad no gubernamental legalmente reconocida en uno o más Estados miembros de la Organización, puede presentar a la Comisión Interamericana de Derechos Humanos peticiones que contengan denuncias o quejas de violación del artículo 7 de la presente Convención por un Estado Parte, y la Comisión las considerará de acuerdo con las normas y los requisitos de procedimiento para la presentación y consideración de peticiones estipulados en la Convención Americana sobre Derechos Humanos y en el Estatuto y el Reglamento de la Comisión Interamericana de Derechos Humanos (Organización de los Estados Americanos-OEA-(1994).

3 Ver el párrafo 57 de la Sentencia de Fondo, Reparaciones y Costas de 2009. Este último fundamento ha sido el prevalente en diversos pronunciamientos de la Corte Interamericana y es el actual, sus bases se encuentra magníficamente explicadas en el voto razonado del Juez García Ramírez en el caso Penal Miguel Castro que se estudiará más adelante, quien aclaró que la protección de los derechos por medio de la vía de denuncias individuales no resulta completa, si debe circunscribirse únicamente a la competencia de la Comisión Interamericana de Derechos Humanos, de manera que el artículo 12 de la Convención debe ser entendido hacia su efectividad, esto es, que incluye la Competencia de Comisión y Corte, de lo contrario no tiene relevancia someter lo estipulado al sistema de peticiones individuales, que como sistema es precisamente integrado, comprende tanto los trámites ante la
Cinco son las sentencias hito emitidas por la Corte Interamericana de Derechos Humanos: caso Penal Castro contra Perú de 2006, Campo Algodonero 2009, las Dos Erres" contra Guatemala 2009, Fernández Ortega contra México, y Valentina Rosendo Cantú contra México, estas últimas de 2010.

En el primero de ellos, Penal Castro contra Perú (2006), se cometieron actos de violencia sexual contra mujeres que se encontraban en custodia y cuidado del Estado. En este fallo se analizaron las obligaciones del Estado en relación con la convención de Belem Do Pará y con base en la interpretación integral de los artículos 7, 8.1 y 25 de la Convención de estableció la importancia de la debida diligencia por parte del Estado en el esclarecimiento de los hechos que victimicen a una mujer. Asimismo, por primera vez estableció la Corte la relación existente entre la violencia basada en género y la discriminación.

En la sentencia ya mencionada en párrafos anteriores, del caso conocido " $\mathrm{El}$ Campo Algodonero" el 16 de noviembre de 2009, la Corte Interamericana de Derechos Humanos encontró responsable al Estado mexicano por el homicidio brutal con móvil sexual de tres mujeres en Juá-

Comisión como ante el Tribunal Interamericano (Acosta, 2009). Lo anterior parece claro, lo que no es claro es si la competencia en esos términos nada más está limitada al artículo 7 de la Convención, o por el contrario se extiende a cada una de las cláusulas del tratado, ya que en algunos casos la Comisión se ha abrogado competencia para estipulaciones por fuera de este artículo 7 tal como sucedió en el caso MZ vs Bolivia (citado por Acosta). 
rez. En esta oportunidad la Corte consideró que este homicidio se dio dentro del marco de la violencia basada en género que se vive en esta ciudad desde 1993, por lo que la opinión pública considera se trata del primer caso en el que se llevaron hechos constitutivos de feminicidio ante la Corte Interamericana. Se aclara, dicho término no fue usado por el Tribunal; en su lugar, se utilizó la expresión homicidio de mujeres por razones de género, al referirse únicamente a las tres mujeres asesinadas.

En la misma decisión, se manifestó que la violencia ejercida contra la mujer es una forma de discriminación, y que en el caso concreto la negligencia con la que las autoridades mexicanas investigaron los hechos constituyó también una actuación de discriminación en cuanto al acceso a la Justicia. La impunidad de los delitos cometidos envía el mensaje de que la violencia contra la mujer es tolerada, lo que favorece su perpetuación y la aceptación social del fenómeno, el sentimiento y la sensación de inseguridad en las mujeres, así como una persistente desconfianza de estas en el sistema de Administración de Justicia (CIDH, 2009A).

Este caso ha trascendido más allá de la esfera de las víctimas, alcanzando un impacto generalizado en la medida que permitió visibilizar la situación sistemática de violencia que padecen las mujeres, en especial en Juárez, llamando la atención en que no se trata de un hecho aislado, sino que el asesinato de las víctimas se corresponde con un contexto de discriminación general y, lo que es peor, de aceptación e impunidad frente a actos de violencia ejercidos contra la mujer.

Al respecto, es de anotar que la presencia del caso en la jurisdicción internacional se debe a la movilización y el activismo no solo en México sino en el mundo para luchar contra los feminicidios de Juárez. El proceso fue desde la sociedad civil organizada en torno a la defensa de los derechos de las mujeres y a la lucha generalizada contra el asesinato sistemático de las mismas por razones de género, activismo que ha cobrado la vida de otras mujeres tal como sucedió con Maricela Escobedo Ortiz ${ }^{4}$, nacida en Chihuahua México, quien fue asesinada en diciembre de 2010 mientras protestaba por la impunidad en el asesinato por motivos de género en contra de su hija, y Susana Chávez Castillo5, natural de Juárez, a su vez asesinada y encontrada el día 6 de enero de 2011 envuelta en una bolsa con rastros de haber sido violada y torturada, en razón a su denuncia y lucha por el esclarecimiento de los hechos de Juárez.

El 24 de noviembre de 2009 la Corte Interamericana profiere la sentencia de "las Dos Erres" contra Guatemala. En esta oportunidad en el marco de un conflicto armado interno. Específicamente durante la masacre de 251 habitantes de la parcelación de Las Dos Erres, La Libertad, Departamento de Petén, en diciembre

\footnotetext{
Puede tenerse mayor información en el sitio de noticias http://www.eluniversal.com.mx/notas/731195. html

5 Puede tenerse mayor información en el sitio de noticias: http://www.bbc.co.uk/mundo/noticias/2011/01/110112 mexico juarez susana_chavez_an.shtml
} 
de 1982, se examinaron la violación sexual y el aborto forzado por golpes en el cuerpo de las mujeres que habitaban la parcelación. En este caso se destacó que en Guatemala, durante el conflicto entre 1962 y 1996, la violencia sexual había sido una práctica de Estado, así como la falta de investigación relativa a este hecho sistemático, constituyendo un irrespeto a las obligaciones establecidas en las normas de derecho internacional de los derechos humanos.

Finalmente, en agosto del año 2010 el Tribunal Interamericano profirió las sentencias de los casos Fernández Ortega contra México (2010A) y Valentina Rosendo Cantú contra México (2010B); en ambos se analizaba la violación y tortura de dos mujeres indígenas mexicanas, la primera de ellas de 27años de edad, y la segunda, de 17.

Tienen en común ambos casos las múltiples condiciones de discriminación que concurren en las mujeres víctimas de tales agresiones, puesto que son mujeres indígenas, enfrentadas a miembros del ejército y consecuentemente al aparato estatal mexicano. En ambos procesos fue condenado el Estado mexicano con importantes consideraciones como que la violencia sexual es una forma paradigmática de violencia contra las mujeres; además, aunque ocurra en un solo hecho aislado, es un acto de tortura; igualmente, siempre que ocurra un hecho de violencia basada en género las autoridades deben garantizar la debida diligencia en los procesos y trámites necesarios para esclarecer los hechos y sancionar de manera ejemplar a los perpetradores; finalmente deben evitar la revictimización de la mujer, por lo que su declaración será valorada en razón a su inmenso valor probatorio.

Este proceso se ha dado de igual forma desde la base social, esta vez, desde el activismo de las organizaciones indígenas de México, que contaron con el apoyo de las diferentes organizaciones de nivel global; de hecho no cesó con las providencias sino que posterior a ellas ha hecho el seguimiento a su cumplimiento de manera estricta, poniendo en la opinión pública el debate acerca de la negligencia de las autoridades en los casos de violencia ejercida contra las mujeres, en especial si se trata de violencia sexual contra mujeres indígenas a causa de procesos de militarización de sus territorios.

En estos cinco casos, las víctimas de las violaciones acompañadas de movimientos y organizaciones de derechos humanos, han realizado activismo trasnacional, no solo durante el proceso ante el Sistema Interamericano, sino posterior a ello en búsqueda y exigencia del cumplimiento de las disposiciones de la Corte. Además, han alcanzado un impacto generalizado al entender los derechos vulnerados en cada uno de los hechos llevados ante el Sistema como asuntos comunes a todas las mujeres que viven en lugares en los que reina el narcotráfico, existe conflicto armado o se encuentran reclusas; pero también como específicos de las mujeres de Juárez, Perú y Guatemala, lo que indudablemente evidencia un proceso de ejercicio de la ciudadanía global. 


\section{CONCLUSIONES}

La ciudadanía global es un concepto que desafía no solo la tradicional concepción de ciudadanía nacional, sino la noción de democracia moderna, en la medida que implica el traspaso de los límites espaciales del Estado-nación, desde todos los elementos del mismo, incluso del ordenamiento jurídico, en la medida que el reconocimiento de ciudadanos globales implica la afirmación de derechos supranacionales, asimilados generalmente al sistema Internacional de Derechos Humanos. No obstante, al igual que la ciudadanía local, requiere de la existencia de unos tribunales de justicia, encargados de garantizar el cumplimiento de estos derechos, a través de mecanismos jurídicos coercitivos; así las cosas, no podemos hablar de ciudadanía global plena, sin referirnos al acceso a los tribunales internacionales en condiciones de equidad, para aquellos casos en los que la justicia local sea denegada.

Teniendo como marco esta interpretación, debe examinarse que las mujeres han sido pioneras en reconocerse como sujetas políticas internacionales; al verse limitadas en su ciudadanía nacional, inician, desde finales del siglo XX, el ejercicio global de sus derechos, ya sea abriendo espacios de reflexión en torno a temas de género, presionando para la institucionalización de instrumentos internacionales o generando agendas trasnacionales de temas problemáticos como la violencia basada en género y los derechos sexuales.
Es precisamente este ejercicio continuo el que ha llegado eficazmente a los tribunales de justicia internacional, obteniendo no solo respuestas concretas a casos de violaciones de los derechos humanos de las mujeres, cosa realmente importante, sino que también ha generado precedentes trasnacionales, que permean los ámbitos locales, contribuyendo a la demanda de una ciudadanía plena de las mujeres tanto en lo global como en lo local.

Sin embargo, es necesario mencionar que las dificultades que traen consigo los sistemas de protección de derechos, son también obstáculos en la consolidación de un proceso global de democracia y, por lo tanto, del ejercicio de la ciudadanía, en especial de los grupos identitarios tradicionalmente excluidos como las mujeres. En un Estado que no reconoce la existencia y competencia de tribunales internacionales, no se garantiza la plenitud del derecho a acceder a la justica y, por lo tanto, se limita un aspecto importante de la ciudadanía de sus asociados; además, también lo hacen aquellos Estados que, habiendo adoptado las competencias de estos tribunales, permanecen remisos al cumplimiento de sus disposiciones o lo dilatan injustamente.

Por su parte, el Sistema Interamericano, en relación con los derechos humanos de las mujeres, es especialmente frágil, dada la falta de claridad inicial en relación con la competencia de la Corte Interamericana para conocer de casos de vulneración de la Convención de Belem Do Pará, como puede observarse en el caso de Campo 
Algodonero (2009) en el que, incluso, parte de la defensa estatal se afianza en la excepción preliminar de falta de competencia por tales motivos. Además, si bien existe un profuso desarrollo interpretativo de las obligaciones de los Estados en caso de violación de los derechos humanos de la mujer, también es cierto que puede establecerse en algunos casos la omisión de la Corte de considerar su propio estándar a pesar de concurrir hechos de violencia basada en género, como sucedió en el caso Caballero Delgado y Santana contra Colombia, en el que tras encontrarse testimonio directo que daba cuenta de vejámenes sexuales padecidos por María del Carmen Santana, la Corte consideró que no había prueba suficiente de los hechos que constituían violencia basada en género (Bustamante $\mathcal{E}$ Vásquez, 2011).

En atención a lo anterior, es necesario fortalecer los tribunales internacionales de protección a los derechos humanos, en la medida que solo a partir de su efectividad, puede hablarse de la plenitud del derecho a acceder a la justicia y, consecuentemente, dar un paso relevante para la integración de ciudadanías en el marco de la sociedad globalizada.

\section{REFERENCIAS BIBLIOGRÁFICAS}

Acosta, J. (2009). Alcance de la competencia contenciosa de la CIDH a la luz del artículo 23 de su reglamento. International Law, Revista Colombiana de Derecho Internacional, (Número 14), pp. 107-131, disponible en http://www.javeriana.edu.co/juridicas/pub rev/documents/ 04ALCANCEDELACOMPETENCIA 000.pdf
Astelarra, J. (2002). Democracia, ciudadanía y Sistema Político de Género. PRIGEPP-FLACSO. Buenos Aires: Seminario PRIGEPP-FLACSO.

Bareiro, L. (2009). Democracia/s, ciudadanía y Estado en América Latina en el Siglo XXI. Análisis de Género de los caminos recorridos desde la década de los 80' y futuros posibles. Unidad N. ${ }^{\circ}$ 2: Ciudadanía Máximo estatus. Derechos humanos. Derecho a tenerlos. Buenos Aires: Seminario PRIGEPPFLACSO.

Bauman, Z. (1999). Turistas y vagabundos. En La Globalización Consecuencias Humanas (pp.103133). México D. F., México. Fondo de Cultura Económica de México. Disponible en https:// estudiscritics.files.wordpress.com/2011/02/ la-globalizacion-zigmunt-bauman.pdf.

Beck, U. (2001). Democracia global: La política más allá del Estado-nación. Revista Metapolítica, volumen 5, (Número 20), pp. 66-71. Seminario PRIGEPP-FLACSO, Buenos Aires.

Bonder, G. (2010). Globalización y género. Dimensiones económicas, políticas, culturales y sociales. Tensiones, reacciones y propuestas emergentes en América Latina. Unidad N.$^{\circ} 1$ : Globalización Conceptos y fenómenos, Unidad $\mathrm{N}$. $^{\circ}$ 2: Globalización en América Latina, Unidad N. ${ }^{\circ}$ 3: Orden Global y Orden de Género. Buenos Aires: Seminario PRIGEPP-FLACSO.

Bustamante, D. E Vásquez, P. (2011). La Convención de Belem Do Pará un Balance de su aplicación en la jurisprudencia de la Corte Interamericana, a 16 años de su entrada en vigor. Revista Civilizar, Volumen 11, (Número 20), enero-junio de 2011, pp. 15-36, disponible en http://www.usergioarboleda.edu.co/civilizar/ civilizar20/LA\%20CONVENCI\%C3\%93N\%20 BEL\%C3\%89M.pdf

Castells, M. (s. f.). La globalización truncada de América Latina. Documentos PRIGEPP, 2010.

Organización de los Estados Americanos -OEA- (1994). Convención Interamericana para prevenir, sancionar y erradicar la Violencia 
contra la Mujer. "Convención De Belem Do Para". Washington: OEA.

Comisión Interamericana de Derechos Humanos. (2011). Estándares jurídicos vinculados a la igualdad de género y a los derechos de las mujeres en el Sistema Interamericano de Derechos Humanos; Desarrollo y Aplicación. Disponible en https:// www.oas.org/es/cidh/mujeres/docs/pdf/ ESTANDARES\%20JURIDICOS.pdf.

Corte Interamericana de Derechos Humanos. (2006). Caso Penal Castro contra Perú, Sentencia de Fondo, Reparaciones y Costas. San José, Costa Rica.

Corte Interamericana de Derechos Humanos. (2009A). Caso del Campo Algodonero, Sentencia de Fondo, Reparaciones y Costas. San José, Costa Rica.

Corte Interamericana de Derechos Humanos. (2009B). Caso "Las Dos Erres" contra Guatemala, Sentencia de Fondo, Reparaciones y Costas. San José, Costa Rica.

Corte Interamericana De Derechos Humanos. (2010A). Caso Fernández Ortega contra México, Sentencia de Fondo, reparaciones y Costas. San José, Costa Rica.

Corte Interamericana de Derechos Humanos. (2010B). Caso Resendo Cantú contra México, Sentencia de Fondo, Reparaciones y costas. San José, Costa Rica.

Delgado, R. (2007). Los marcos de acción colectiva y sus implicaciones culturales en la construcción de ciudadanía. Revista Universitas Humanística, (Número 64), julio-diciembre de 2007, pp. 41-66.

Ferrer, A. (1998). La Globalización, la crisis financiera y América Latina. Documentos PRIGEPP, 2010. Argentina: Consejo Latinoamericanos de Ciencias Sociales (CLACSO).

Ferrer, N. (2010). El acceso a la justicia como elemento indispensable de la ciudadanía femenina. Revista Opinión Jurídica, Volumen 9 , (Número 17), enero-junio de 2010, pp. 113-124.
Fries, L. (2000). Los derechos humanos de las mujeres: aportes y desafíos. En Las Fisuras del patriarcado reflexiones sobre el feminismo y el derecho (pp. 45 - 64). Quito, Ecuador: FLACSO. Disponible en: http://www.flacso.org.ec/ docs/safisuras.pdf.

Hurtado, J. (2000). Metodología de investigación holística (3ra Ed.). Caracas, Venezuela: Fundación Sypal.

Mantilla, J. y Uprimny, R. (2009). Violencia de Género y Justicia Constitucional en Colombia. En Justicia desigual. Género y derechos de las víctimas en Colombia. Violencia de género y justicia constitucional en Colombia (pp. 117-163). Bogotá, Colombia. Fondo de Desarrollo de las Naciones Unidas para la MujerUNIFEM.

Marshall, T. H. (1998). Ciudadanía y clase social. En Ciudadanía y Clase Social (pp. 15-82) Editor Tom Bottomore, Madrid, España. Alianza Editorial, disponible en file:///C:/Users/nina. ferrer/Downloads/Ciudadania_y_Clase_Social_ T.H.Marshall.pdf.

Pateman, C. (2002). Democratization and Women's Self-Government. Unidad No. 2: Ciudadanía Máximo estatus. Derechos humanos. Derecho a tenerlos. Buenos Aires: Seminario PRIGEPPFLACSO. Documentos PRIGEPP, 2009.

Sassen, S. (s. f.). Para que funcione la economía global: el papel de los estados nacionales y los organismos privados. Unidad N. ${ }^{\circ}$ : Globalización conceptos y fenómenos, Unidad N. 2 : Globalización en América Latina, Unidad N. ${ }^{\circ}$ 3: Orden global y orden de género. Buenos Aires: Seminario PRIGEPP-FLACSO. Documentos PRIGEPP, 2010

Stromquist, N. (2009). Teorizando la ciudadanía global: discursos, desafíos e implicaciones para la educación. Revista Interamericana de Educación para la Democracia, Volumen 2, (Número 1), pp. 6-31, recuperado de http://www.ried-ijed.org/spanish/articulo. php?idRevista $=9$ EidArticulo $=26$ 
Vargas, V. (2001). Ciudadanías globales y sociedades civiles globales. Pistas para el análisis. Revista Utopías, Nuestra Bandera: Revista de Debate Político. N. ${ }^{\circ}$ 187, 99-113.
Villelas, M. (2010). La violencia sexual como arma de guerra. En Quaderns de Construcció de Pau, (Número 15), pp. 3-15, Agencia Catalana de Cooperación para el Desarrollo, disponible en http://escolapau.uab.es/img/qcp/violencia sexual_guerra.pdf. 
